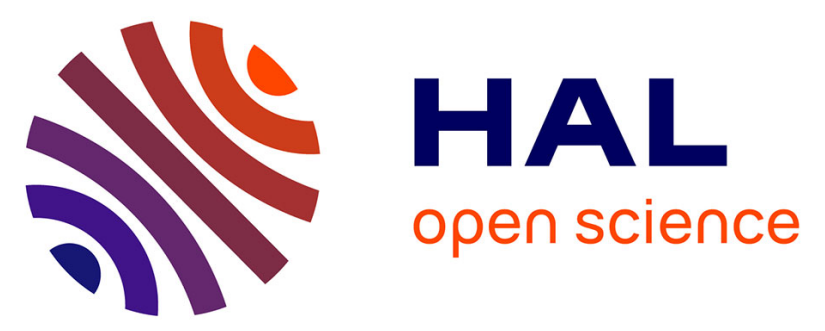

\title{
Reliable Protocol for Shear Wave Elastography of Lower Limb Muscles at Rest and During Passive Stretching.
} Guillaume Dubois, Walid Kheireddine, Claudio Vergari, Dominique Bonneau, Patricia Thoreux, Philippe Rouch, Mickael Tanter, Jean-Luc Gennisson, Wafa Skalli

\section{To cite this version:}

Guillaume Dubois, Walid Kheireddine, Claudio Vergari, Dominique Bonneau, Patricia Thoreux, et al.. Reliable Protocol for Shear Wave Elastography of Lower Limb Muscles at Rest and During Passive Stretching.. Ultrasound in Medicine \& Biology, 2015, 41 (9), pp.2284-2291. 10.1016/j.ultrasmedbio.2015.04.020 . hal-02494020

\author{
HAL Id: hal-02494020 \\ https://hal.science/hal-02494020
}

Submitted on 28 Feb 2020

HAL is a multi-disciplinary open access archive for the deposit and dissemination of scientific research documents, whether they are published or not. The documents may come from teaching and research institutions in France or abroad, or from public or private research centers.
L'archive ouverte pluridisciplinaire HAL, est destinée au dépôt et à la diffusion de documents scientifiques de niveau recherche, publiés ou non, émanant des établissements d'enseignement et de recherche français ou étrangers, des laboratoires publics ou privés. 


\title{
RELIABLE PROTOCOL FOR SHEAR WAVE ELASTOGRAPHY OF LOWER LIMB MUSCLES AT REST AND DURING PASSIVE STRETCHING
}

\author{
Guillaume Dubois, ${ }^{*}$ Walid Kheireddine, ${ }^{*}$ Claudio Vergari, ${ }^{*}$ Dominique Bonneau,* \\ Patricia Thoreux,${ }^{* \dagger}$ Philippe Rouch, ${ }^{*}$ Mickael Tanter, ${ }^{\ddagger}$ Jean-Luc Gennisson, ${ }^{\ddagger}$ \\ and WAFA SKALLI* \\ * LBM/Institut de Biomécanique Humaine Georges Charpark, Arts et Métiers ParisTech, 151 Boulevard de l'Hôpital, Paris \\ 75013, France; ${ }^{\dagger}$ Universite Paris XIII, Paris, France; and ${ }^{\ddagger}$ Institut Langevin, Laboratoire Ondes et Acoustique, CNRS UMR \\ 7587, ESPCI ParisTech, PSL, INSERM ERL U979, Paris, France
}

\begin{abstract}
Development of shear wave elastography gave access to non-invasive muscle stiffness assessment in vivo. The aim of the present study was to define a measurement protocol to be used in clinical routine for quantifying the shear modulus of lower limb muscles. Four positions were defined to evaluate shear modulus in 10 healthy subjects: parallel to the fibers, in the anterior and posterior aspects of the lower limb, at rest and during passive stretching. Reliability was first evaluated on two muscles by three operators; these measurements were repeated six times. Then, measurement reliability was compared in 11 muscles by two operators; these measurements were repeated three times. Reproducibility of shear modulus was $0.48 \mathrm{kPa}$ and repeatability was $0.41 \mathrm{kPa}$, with all muscles pooled. Position did not significantly influence reliability. Shear wave elastography appeared to be an appropriate and reliable tool to evaluate the shear modulus of lower limb muscles with the proposed protocol. (E-mail: guillaume.dubois@ensam.eu)
\end{abstract}

Key Words: Elastography, Muscle, Shear modulus, Reliability, Lower limb.

\section{INTRODUCTION}

In vivo assessment of muscle properties is a challenge that needs to be met to quantify neuromuscular diseases or evaluate their treatment. A quick-release method for evaluation of the elasticity of the muscle-tendon unit was investigated by Cornu et al. (2001), but this technique cannot isolate the behavior of one muscle. In addition, it is technically difficult to use in subjects with high joint stiffness or bone deformities. Stiffness of the gastrocnemius muscle-tendon unit of the calf has been evaluated by numerical optimization of the measurement of passive ankle torque depending on ankle angle (Hoang et al. 2005; Nordez et al. 2010). This elegant method had low reliability for some parameters and cannot be used to evaluate only one muscle. Magnetic resonance elastography (MRE) has also been used to evaluate muscle stiffness as shear modulus in three dimensions (Basford et al. 2002; Bensamoun et al. 2007; Ringleb et al. 2007). However, the measurement can only be performed with the patient lying down, and the acquisition cost remains a limitation. Despite the small number of subjects, stiffness in resting muscles appeared lower in control subjects than in patients with neuromuscular dysfunction (Basford et al. 2002). This difference was highlighted in vitro by comparing the stiffness of stretched muscle fiber bundles during a tensile test (Smith et al. 2011).

Shear wave elastography (SWE) has been used to assess the mechanical properties of soft tissue for about 10 years. The concept of SWE imaging (SWEI) using acoustic radiation force was proposed by Sarvazyan et al. (1998). More recently, Bercoff et al. (2004) coupled this concept with ultrafast ultrasound imaging to provide a specific technique called supersonic shear imaging. This technique provides a quick measurement of the muscle shear modulus using a standard ultrasonic probe (Gennisson et al. 2010). However, there is lack of consensus in the literature on the technical aspects of acquiring elastographic measurements. This has resulted 
in large variability between studies depending on the measurement technique (subject position, measurement position, at rest or during contraction, etc.).

Kot et al. (2012) found that size of the region of interest (ROI) and probe pressure influence elastography measurement. Moreover, subject position affects the measurement: an increase in shear modulus was observed when muscle was passively stretched, both in vitro (Koo et al. 2013; Maïsetti et al. 2012; Shinohara et al. 2010) and ex vivo (Eby et al. 2013). Maïsetti et al. (2012) and Hug et al. (2013) determined in vivo the slack length of the muscle, corresponding to a range of motion within which the muscle does not produce any passive force and shear modulus is constant.

Clinically, SWE was recently used to evaluate muscles in a subject with patellofemoral pain syndrome by Botanlioglu et al. (2013), who reported that the vastus medialis obliquus was less stiff during contraction in patients with pathology than in healthy patients, but they did not perform a reliability study.

Lacourpaille et al. (2012) evaluated the reliability of this technique in nine muscles and proposed standardizing the muscle length by controlling joint angles, which were chosen to leave the muscle as slack as possible. These positions seemed to improve reliability because of the range of muscle slack length, which allowed the shear modulus to remain constant under small angle variations between joints.

Although measurements in muscle at rest provide an interesting physiologic baseline, the stiffness of contracted or stretched muscle is more likely to differ between healthy and pathologic muscles (Botanlioglu et al. 2013); indeed, most clinical qualitative examinations are based on the response of the muscle to external mechanical solicitation. Voluntary contraction, however, is difficult to reliably reproduce and maintain during the measurement. Fatigue and trembling can occur rapidly, even in isometric contraction, thus negatively affecting measurement reliability. Passive stretching could represent an interesting alternative, but to our knowledge no study has characterized measurement reliability during passive stretching.

The aim of the present study was to define a measurement protocol to quantify the shear modulus of 11 muscles of the lower limb, both at rest and during passive stretching, and to quantify inter-operator reproducibility and intra-operator repeatability.

\section{METHODS}

\section{Subjects}

Ten subjects (age: $25.5 \pm 2.8$ y, height: $176 \pm 11.0$ $\mathrm{cm}$, weight: $68 \pm 13.3 \mathrm{~kg}$, body mass index [BMI]: 21.7 $\pm 2.0 \mathrm{~kg} / \mathrm{m}$ ) with no documented muscular pathology gave their written consent to participate in this protocol (approved by the Institutional Ethics Committee, CPP 06036, Paris).

\section{Shear wave elastography measurement}

The principle underlying SWE using supersonic shear wave imaging (SSI) was previously described (Bercoff et al. 2004). Briefly, a cylindrical shear wave front is generated in the tissue by successively focusing ultrasonic pushing beams at different depths. Then, with very high frame rate imaging (up to 20,000 images), a movie of the shear wave propagating is recorded in an imaging plane parallel to the main axis of the cylinder, which is parallel to the ultrasound axis. In this plane, Bmode images and shear wave velocity movies are acquired. Last, the local shear wave speed is retrieved from a time-of-flight algorithm.

Measurements were performed with an Aixplorer ultrasound scanner (Version 4.2, Supersonic Imagine, Aix-en-Provence, France) driving a 4- to $15-\mathrm{MHz}$ ultrasonic probe (SL15-4, 256 elements, pitch $0.2 \mathrm{~mm}$ ). Acquisitions were performed in general mode with the following SWE settings throughout the whole study: penetration mode, tissue tuner at $1540 \mathrm{~m} / \mathrm{s}$, middle persistence, smoothing 5, SuperRes 2.

Muscle is highly anisotropic; therefore, the shear wave velocity depends on the probe orientation relative to the muscle fibers (Gennisson et al. 2010; Lee et al. 2012; Rouze et al. 2013; Wang et al. 2013). Thus, acquisitions were performed with the probe in a plane parallel to the muscle fibers and perpendicular to the skin. This orientation was determined when several fibers were continuously visible on the B-mode image (Fig. 1). When the ultrasonic probe is properly aligned with the muscle fibers, shear wave speed $V_{\mathrm{s} / /}$ is directly linked to the shear modulus $\mu_{/ /}$in the longitudinal direction by (Gennisson et al. 2003; Royer et al. 2011)

$$
\mu_{/ /}=\rho \cdot V_{\mathrm{s} / /}^{2} \quad \text { with } \rho=1000 \mathrm{~kg} / \mathrm{m}^{3}
$$

For muscles with several bellies, such as the rectus femoris, only one belly was measured. For each measurement, a sequence of 10 continuous images was recorded in $10 \mathrm{~s}$ films. Shear modulus images were then processed using custom software developed in MATLAB (The MathWorks, Natick, MA, USA) for rapid and semiautomatic processing of all 1020 images. The ROI was defined as a rectangle (which is adapted to thin and long muscle):

1. The operator selected an ROI on the first image; the ROI was then automatically tracked on subsequent images using a difference-based algorithm. The ROI was defined in the square region of shear modulus measurement as the largest square between fasciae (Fig. 1). 


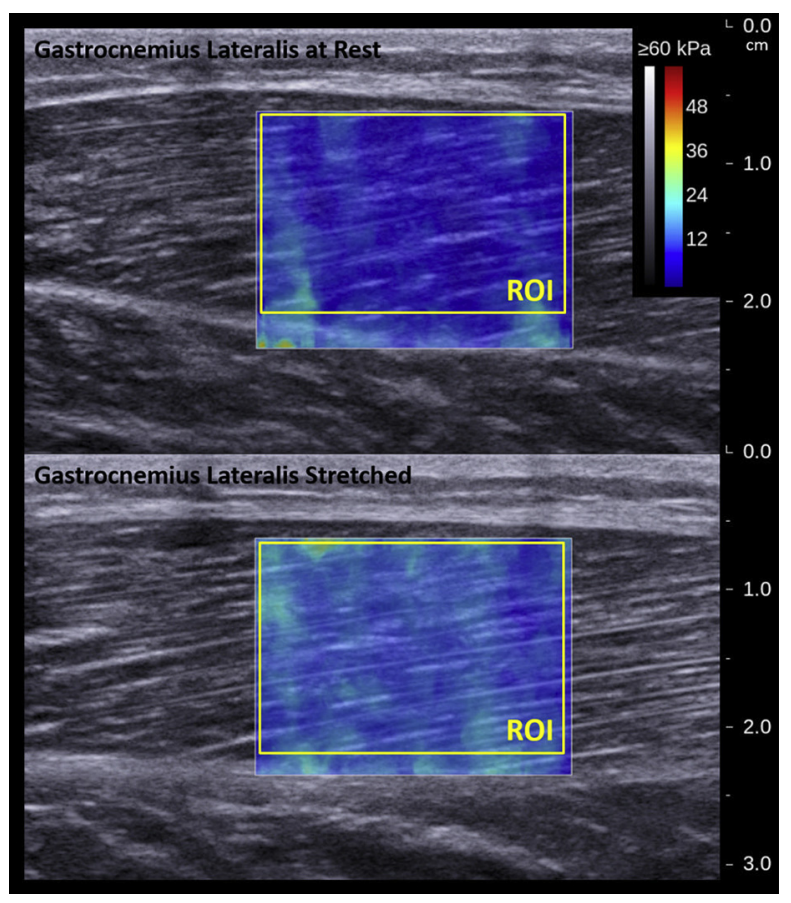

Fig. 1. Shear modulus assessment using elastography in rested and stretched gastrocnemius lateralis muscle. ROI = region of interest.

2. In the ROI of each image, the RGB value of each pixel color was converted to a shear modulus value, accounting for color chart opacity, according to the color bar, which is composed of 221 distinct colors.

3. Muscle shear modulus was computed as the mean shear modulus of the ROI in all images.

\section{Protocols}

Measurements were performed by operators familiar with muscle elastography; the operator replaced the probe after each measurement. A large amount of gel was applied between the probe and the skin to limit tissue deformation induced by the operator (Kot et al. 2012).

\section{Positions}

SWE was performed both at rest and during passive stretching in the 11 main muscles involved in knee joint motion and plantar flexion of the foot: biceps femoris (BF), gracilis (GRA), rectus femoris (RF), sartorius (SAR), semimembranosus (SM), semitendinosus (ST), vastus lateralis (VL), vastus medialis (VM), gastrocnemius medialis $(\mathrm{GM})$, gastrocnemius lateralis (GL) and soleus (SOL).

Four positions were defined to measure the muscles of the anterior and posterior aspects of the limb at rest and during passive stretching:

1. RF, VM and VL passively stretched: The subject lay supine on the table with the calves and the knees hanging outside the table. The subject was asked to remain in a comfortable and relaxing position (Fig. 2a).

2. RF, VM and VL at rest: The subject sat upright on the table with the heels outside the table; the angle between the trunk and thighs was approximately $90^{\circ}$ (Fig. 2b).

3. BF, ST, SM, GRA, SAR, GL, GM and SOL passively stretched: The subject lay prone on the table with the feet outside the table (Fig. 2c). This position stretched the muscles of the posterior aspect of the thigh while also leaving the subject in a comfortable position which could be easily assumed by patients with pathology as well.

4. BF, ST, SM, GRA, SAR, GL, GM and SOL at rest: The subject lay prone on the table with an angle of approximately $90^{\circ}$ between the legs and thighs (Fig. 2d). For both protocols, the same order of positions $(1,2,3,4)$ was used.

\section{Measurement location}

To standardize the measurement location, landmarks were drawn on the subject's skin. For the anterior and posterior thigh muscles, the anterosuperior iliac spine and the apex of the patella were identified by palpation, and the inferior third of their distance was marked. For the posterior leg muscles, proximal and distal limits of the fibula were identified by palpation, and the superior third of the distance was marked.

\section{Complete protocol}

The complete protocol was used to evaluate intraoperator measurement repeatability and inter-operator reproducibility, particularly as related to the proposed subject positions. Measurements were performed by three operators and repeated six times in each of the previously defined positions. This complete protocol was used on only one muscle of the thigh and one of the calf (VM and GL). It was assumed that the repeatability of these two muscles was representative of the repeatability of measurements of the lower limb. This protocol lasted about $75 \min (3$ operators $\times 6$ measurements $\times 2$ positions $\times 2$ muscles $\times \sim 1$ min per measurement).

For measurements on patients in the supine position, position 1 (Fig. 2a), anterior muscles were passively stretched, and subjects reported being uncomfortable after a few minutes. This was accompanied by a measurable increase in shear modulus, which was observed during preliminary tests. A similar phenomenon was previously observed by Lacourpaille et al. (2012), but such an increase in modulus was eliminated by asking the subject to freely stretch out and walk between series of measurements. 

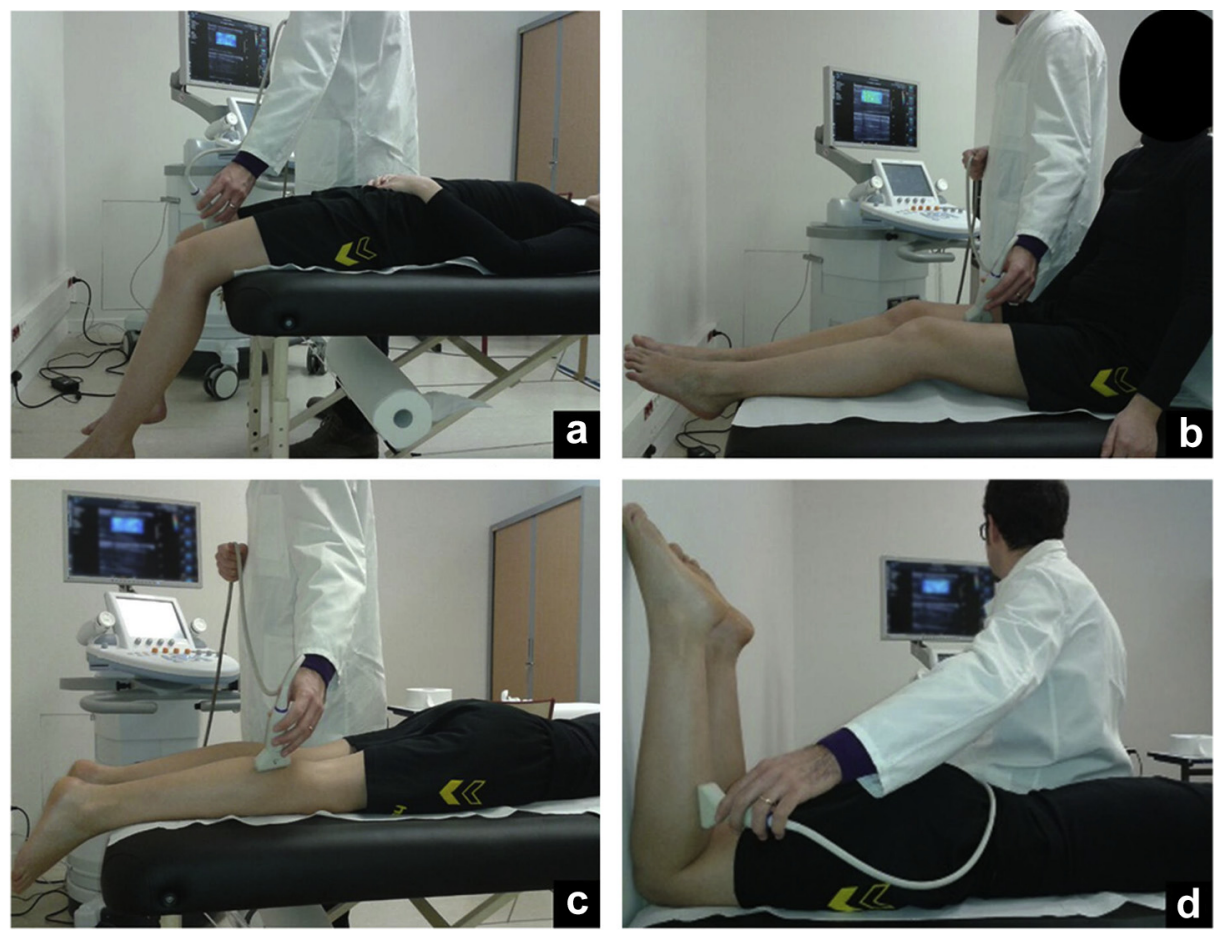

Fig. 2. Measurement positions of shear modulus in rested and stretched muscles of the thigh and calf. BF $=$ biceps femoris; GL = gastrocnemius lateralis; GM = gastrocnemius medialis; GRA = gracilis; RF = rectus femoris; $\mathrm{SAR}=$ sartorius; $\mathrm{SM}=$ semimembranosus; $\mathrm{SOL}=$ soleus; $\mathrm{ST}=$ semitendinosus; $\mathrm{VL}=$ vastus lateralis; $\mathrm{VM}=$ vastus medialis.

\section{Reduced protocol}

Once the effects of operator and position on the measurement were determined, a reduced protocol was used to compare the reliability of measurements of the remaining muscles and to evaluate stiffness at rest and during passive stretching. Smaller numbers of operators and repetitions were used: measurements were performed by two operators and were repeated three times. This reduced protocol including all muscles took about 90 min because of the increased number of muscles.

In the remainder of this article, $\mathrm{R}$ and $\mathrm{S}$ subscripts indicate muscles at rest and stretched (e.g., $\mathrm{BF}_{\mathrm{S}}=$ biceps femoris stretched).

\section{Statistical analysis}

Inter-operator reproducibility and intra-operator repeatability of shear modulus measurement were determined to estimate the uncertainty of measurement as recommended by the ISO 5725 Standard for evaluation of the reliability (in terms of repeatability and reproducibility) of measurement methods. Reliability was evaluated for each muscle in both positions. The intra-class correlation coefficient (ICC [2,1]) (Hopkins 2000) was also computed, and a Bland-Altman plot was created for the complete protocol.

Differences between shear moduli of rested and stretched muscles were evaluated using the Wilcoxon signed-rank test, with the level of significance set at 0.05 .
To evaluate the consistency of the number of subjects, a power analysis was performed: a cohort of 10 subjects gave an overall statistical power of $79 \%(\alpha$-level $=0.05)$.

\section{RESULTS}

\section{Complete protocol}

Shear modulus ranged from $3.9 \pm 0.6 \mathrm{kPa}$ for GL to $4.0 \pm 0.7 \mathrm{kPa}$ for $\mathrm{VM}$ at rest and from $5.6 \pm 1.3 \mathrm{kPa}$ for GL to $7.6 \pm 1.4 \mathrm{kPa}$ for $\mathrm{VM}$ under passive stretching. Inter-operator reproducibility of the shear modulus (Table 1) ranged from $0.3 \mathrm{kPa}$ for $\mathrm{GL}_{\mathrm{R}}$ to $0.7 \mathrm{kPa}$ for $\mathrm{VM}_{\mathrm{S}}$, and intra-operator repeatability ranged from 0.3 $\mathrm{kPa}$ for $\mathrm{GL}_{\mathrm{R}}$ to $0.6 \mathrm{kPa}$ for $\mathrm{GL}_{\mathrm{S}}$. The inter-operator coefficient of variation $(\mathrm{CV})$ ranged from $8 \%$ for $\mathrm{GL}_{\mathrm{R}}$ to $11 \%$ for $\mathrm{GLM}_{\mathrm{S}}$, and the intra-operator CV ranged from $7 \%$ for $\mathrm{GL}_{\mathrm{R}}$ to $8 \%$ for $\mathrm{VM}_{\mathrm{R}}$.

Inter-operator ICCs ranged from 0.87 for $\mathrm{GL}_{\mathrm{S}}$ to 0.91 for $\mathrm{GL}_{\mathrm{R}}$, whereas intra-operator ICCs ranged from 0.91 for $\mathrm{VM}_{\mathrm{R}}$ to 0.94 for $\mathrm{GL}_{\mathrm{S}}$. Position was found to have a significant effect on shear modulus for both muscles (VM: $p<0.002$, GL: $p<0.002$ ).

For all muscles pooled, inter-operator reproducibility was $0.5 \mathrm{kPa}(\mathrm{CV}=9 \%)$ and intra-operator repeatability was $0.4 \mathrm{kPa}(\mathrm{CV}=8 \%)$. Thus, the 95\% confidence interval (CI) was lower than $0.8 \mathrm{kPa}$ for $\mathrm{VM}_{\mathrm{R}}$ and $\mathrm{GL}_{\mathrm{R}}$ and lower than $1.4 \mathrm{kPa}$ for $\mathrm{VM}_{\mathrm{S}}$ and $\mathrm{GL}_{\mathrm{S}}$. 
Table 1. Inter- and intra-operator reliability of the shear modulus measurement computed: 10 subjects, three operators, six measures each, rested and stretched VM and GL

\begin{tabular}{llllll}
\hline & $\mathrm{VM}_{\mathrm{R}}$ & $\mathrm{VM}_{\mathrm{S}}$ & $\mathrm{GL}_{\mathrm{R}}$ & $\mathrm{GL}_{\mathrm{S}}$ & Globality \\
\hline Mean (kPa) & 4.0 & 7.6 & 3.9 & 5.6 & 5.3 \\
Standard deviation (kPa) & 0.7 & 1.4 & 0.6 & 1.3 & 1.8 \\
Inter-operator reproducibility $(\mathrm{kPa})$ & 0.4 & 0.7 & 0.3 & 0.6 & 0.5 \\
$\quad$ Coefficient of variation $(\%)$ & 9 & 9 & 8 & 11 & 9 \\
Intra-class coefficient & 0.88 & 0.90 & 0.91 & 0.87 & \\
Intra-operator repeatability (kPa) & 0.3 & 0.6 & 0.3 & 0.5 & 0.4 \\
$\quad$ Coefficient of variation $(\%)$ & 8 & 8 & 7 & 8 & 8 \\
Intra-class coefficient & 0.91 & 0.92 & 0.92 & 0.94 & \\
\hline
\end{tabular}

$\mathrm{GL}=$ gastrocnemius lateralis; $\mathrm{VM}=$ vastus medialis; subscript $\mathrm{R}=$ rested; subscript $\mathrm{S}=$ stretched.

Bland-Altman diagrams (Fig. 3) indicate the level of agreement between the measured values and the dispersion of the measurements.

The $95 \%$ CI was lower than $0.8 \mathrm{kPa}$ when muscle was at rest and lower than $1.4 \mathrm{kPa}$ when muscle was stretched.

\section{Reduced protocol}

Shear modulus ranged from $3.9 \pm 0.6 \mathrm{kPa}$ for $\mathrm{VM}$ to $6.6 \pm 1.4 \mathrm{kPa}$ for SOL at rest, and from $5.1 \pm 1.4 \mathrm{kPa}$ for ST to $13.9 \pm 3.9 \mathrm{kPa}$ for $\mathrm{RF}$ under passive stretching.

Inter-operator reproducibility ranged from $0.4 \mathrm{kPa}$ for $\mathrm{GM}_{\mathrm{R}}$ to $1.6 \mathrm{kPa}$ for $\mathrm{BF}_{\mathrm{S}}$, whereas intra-operator repeatability ranged from $0.2 \mathrm{kPa}$ for $\mathrm{VM}_{\mathrm{R}}$ to $1.4 \mathrm{kPa}$ for $\mathrm{BF}_{\mathrm{S}}$ (Table 2). The inter-operator $\mathrm{CV}$ ranged from $7 \%$ for $\mathrm{VM}_{\mathrm{S}}$ to $24 \%$ for $\mathrm{ST}_{\mathrm{S}}$, and the intra-operator $\mathrm{CV}$ ranged from $5 \%$ for $\mathrm{GM}_{\mathrm{S}}$ to $15 \%$ for $\mathrm{SOL}_{\mathrm{S}}$. Position was found to have a significant effect on shear modulus for all muscles $(p<0.001)$ except the SOL, the $p$ value of which was slightly over the significance threshold $(p=0.059)$ (Table 2). For all muscles pooled, interoperator reproducibility was $1.1 \mathrm{kPa}(\mathrm{CV}=17 \%)$ and intra-operator repeatability was $0.8 \mathrm{kPa}(\mathrm{CV}=12 \%)$.

At rest, six muscles had a $95 \%$ CI between 0.6 and $1.4 \mathrm{kPa}$ : RF, SAR, VL, VM, GL and GM. Five muscles had a $95 \%$ CI between 1.6 and $2.6 \mathrm{kPa}$ : BF, GRA, SM, ST and SOL.

Measurement in a stretched position was slightly less reliable, but not significantly so (Wilcoxon signed rank test: $p=0.83$ ). Three muscles had a $95 \% \mathrm{CI}<1.4$ kPa: VM, GL and GM. Six muscles had a $95 \%$ CI between 2.0 and $2.6 \mathrm{kPa}$ : RF, SAR, SM, ST, VL and SOL. Two muscles had a $95 \%$ CI between 3.0 and $3.2 \mathrm{kPa}$ : BF and GRA.

\section{DISCUSSION}

The aim of the present study was to evaluate the inter-operator reproducibility and intra-operator repeat- ability of a measurement protocol used to assess the shear modulus of the lower limb muscles at rest and during passive stretching. Repeatability and reproducibility were determined in a two-phase study; in the first phase, it was ascertained for two muscles (vastus medialis and gastrocnemius lateralis) that operator or patient position had no effect on measurement reliability. In the second phase, measurement reliability was confirmed in a larger number of muscles. This second phase was necessary because muscle size and anatomic position (e.g., muscle depth) can affect SWE measurements.

The small differences in reproducibility between the complete and reduced protocols can be explained by the smaller numbers of operators and measurement repetitions, which decreased the degrees of freedom (from 17 to 5) for each muscle. The complete protocol is more representative of the actual reliability of measurements because of the larger number of measures. This complete protocol could not be applied to all muscles as it was very time consuming. However, it was necessary to determine that there was no operator effect on the measurements (which were performed by experienced operators) and that the same reliability could be obtained in all positions (Wilcoxon signed rank test: $p=0.83$ ); once that was demonstrated, the reduced protocol allowed estimation of the muscledependent uncertainty in the rest of the muscle.

A choice was made not to strictly control joint angles; subjects were asked to assume the given positions and were instructed to be comfortable. The main objective of the present study was to evaluate the reliability of a protocol as compatible with clinical routine. We therefore chose to let the patient assume a natural posture which would be more easily reproduced during a routine clinical exam, unlike fixed-angle postures which might require an external fixture to be reproduced. It is interesting that intra-subject variability was relatively small $(8 \%)$ even if joint angles were not controlled, thus confirming that small variations in joint angle produce small variations in muscle stiffness.

For the same reason, the number of measurements was kept to a minimum in the reduced protocol (i.e., three measurements in the longitudinal direction). Performing measurements in the other direction with strict control of measurement orientation would allow more thorough characterization of the tissue (Gennisson et al. 2010; Lee et al. 2012; Rouze et al. 2013; Wang et al. 2013), but would significantly increase measurement time and complexity.

Shear modulus was significantly higher when muscle was stretched than at rest $(p<0.05)$ for all measured muscles except the soleus $(p=0.059)$. This result was expected, given the mechanical non-linear behavior of the muscle, and is in accordance with previous studies by Maïsetti et al. (2012) and Hug et al. (2013), who observed an increase in muscle shear modulus during passive 


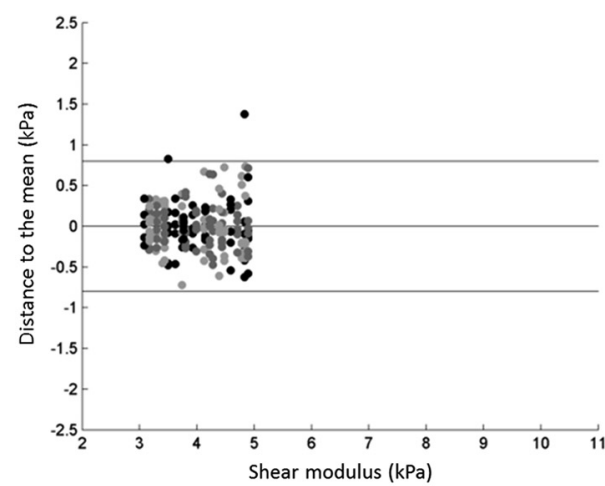

Bland-Altman diagram for VM at rest.

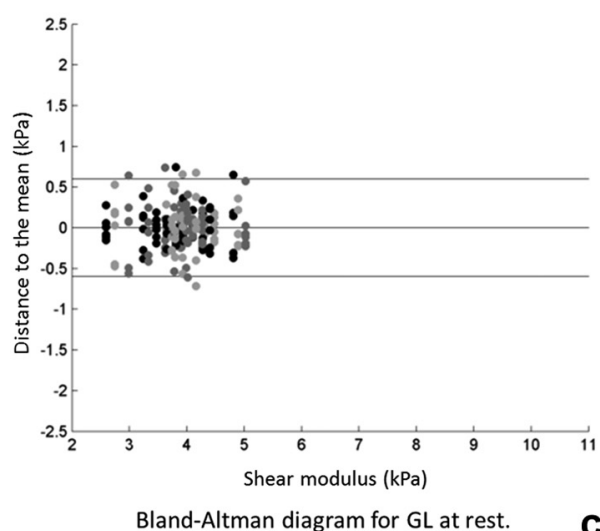

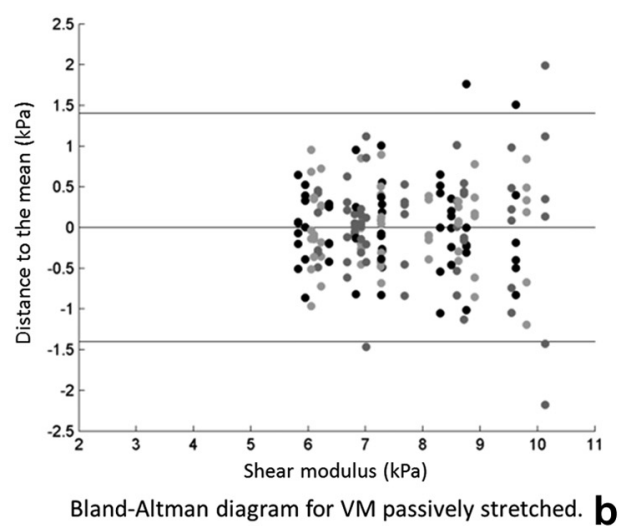

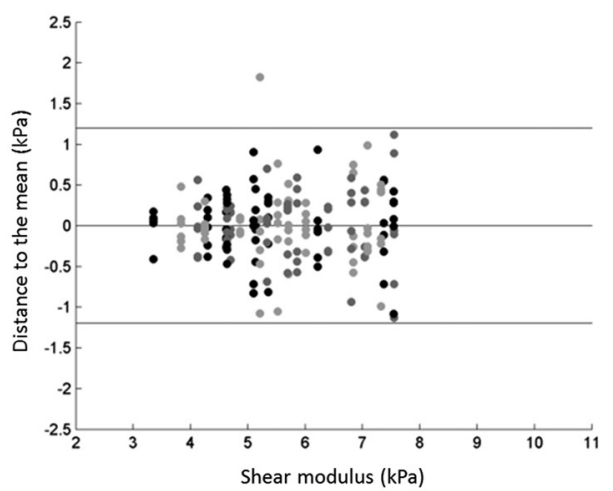

Bland-Altman diagram for GL passively stretched.

Fig. 3. Bland-Altman diagrams for the complete protocol applied in rested and stretched VM and GL. Each operator is represented by a different gray-scale point. $\mathrm{GL}=$ gastrocnemius lateralis; $\mathrm{VM}=$ vastus medialis.

stretching once the slack length threshold was passed. The soleus is not involved in knee motion; the nonsignificant difference confirmed that, although the ankle joint angle was not strictly controlled, muscle stiffness varied little between positions.

On the anterior part of the thigh, the shear modulus of the RF increased more than that of the VM or VL. The RF is a bi-articular muscle: its origin is on the pelvis, and its insertion is on the quadriceps tendon. The VM and VL are mono-articular muscles: their origins are on the femur, and their insertions are on the quadriceps tendon. Thus, the RF was stretched more in position 1 (corresponding to a supine position with an angle of approximately $90^{\circ}$ between leg and thigh).

Reliability of measurements of the SOL and BF was lower than that of other muscles. Difficulty was encountered with the elastographic device in measuring SOL shear wave velocity for some subjects who had thick GL and GM. To improve reliability in such deep muscles (as the SOL), measurements could be performed with a lower-frequency probe, which suffers less from attenuation. Vastus intermedialis muscle was excluded from the study for the same reason: it is a deep muscle, and a reliable signal could not be obtained in it. This is a limitation of the technique.

With respect to the BF, measurement reliability was affected by the fact that this muscle is one of the thinnest of the thigh. The small thickness makes it difficult to distinguish both heads; therefore, positioning of the probe parallel to the fibers was not always straightforward.

Intra-operator muscle reliability was less than $8 \%$ and inter-operator muscle reliability was less than $11 \%$ for the complete protocol. These results are close to those reported by Lacourpaille et al. (2012), who obtained an intra-operator repeatability lower than $6 \%$ and interoperator reliability lower than $11.5 \%$. Values measured by Lacourpaille et al. (2012) were lower by about $1 \mathrm{kPa}$ than those obtained at rest in this study (GM: 2.99 vs. 4.7, VL: 3.26 vs. 4.5 , RF: 3.23 vs. 4.1 ). Both studies were performed on a homogeneous population of healthy young subjects. The values can be explained by differences in participation in sports or differences in fat infiltration in muscles. Larger cohorts are needed to properly determine reference values for different populations. A BMI indicating overweight or obesity should mean increased fat infiltration and should probably increase 
Table 2. Inter- and intra-operator reliability of the shear modulus measurements computed (10 subjects, two operators, three measures each) for rested and stretched BF, GRA, RF, SAR, SM, ST, VL, VM, GM, GL and SOL

\begin{tabular}{|c|c|c|c|c|c|c|c|c|c|c|c|}
\hline & \multicolumn{11}{|c|}{ Rested muscle } \\
\hline & \multicolumn{8}{|c|}{ Thigh } & \multicolumn{3}{|c|}{ Leg } \\
\hline & $\mathrm{BF}$ & GRA & $\mathrm{RF}$ & SAR & SM & ST & VL & VM & GL & GM & SOL \\
\hline Mean (kPa) & 5.6 & 6.0 & 4.1 & 5.3 & 5.3 & 4.2 & 4.5 & 3.9 & 4.5 & 4.7 & 6.6 \\
\hline Standard deviation $(\mathrm{kPa})$ & 1.4 & 1.7 & 0.6 & 1.1 & 1.5 & 1.0 & 1.0 & 0.6 & 0.9 & 0.7 & 1.4 \\
\hline Intra-operator repeatability $(\mathrm{kPa})$ & 1.0 & 1.0 & 0.5 & 0.7 & 0.8 & 0.8 & 0.6 & 0.6 & 0.7 & 0.4 & 1.3 \\
\hline Coefficient of variation $(\%)$ & 18 & 16 & 12 & 17 & 15 & 20 & 12 & 14 & 15 & 9 & 20 \\
\hline Inter-operator reproducibility $(\mathrm{kPa})$ & 0.5 & 0.5 & 0.3 & 0.5 & 0.6 & 0.3 & 0.4 & 0.2 & 0.5 & 0.3 & 0.7 \\
\hline \multirow{4}{*}{ Coefficient of variation $(\%)$} & 10 & 8 & 7 & 9 & 11 & 7 & 9 & 6 & 11 & 6 & 11 \\
\hline & \multicolumn{11}{|c|}{ Stretched muscle } \\
\hline & \multicolumn{8}{|c|}{ Thigh } & \multicolumn{3}{|c|}{ Leg } \\
\hline & $\mathrm{BF}$ & GRA & $\mathrm{RF}$ & SAR & SM & ST & $\mathrm{VL}$ & VM & GL & GM & SOL \\
\hline Mean $(\mathrm{kPa})$ & 10.1 & 8.9 & 13.9 & 7.6 & 8.7 & 5.1 & 6.3 & 7.3 & 5.8 & 6.2 & 7.0 \\
\hline Standard deviation $(\mathrm{kPa})$ & 2.1 & 1.9 & 3.9 & 1.4 & 1.5 & 1.4 & 1.6 & 0.9 & 0.8 & 1.2 & 1.7 \\
\hline Intra-operator repeatability $(\mathrm{kPa})$ & 1.6 & 1.5 & 1.2 & 1.2 & 1.0 & 1.2 & 1.1 & 0.5 & 0.5 & 0.5 & 1.3 \\
\hline Coefficient of variation (\%) & 16 & 17 & 8 & 15 & 12 & 24 & 18 & 7 & 9 & 9 & 19 \\
\hline Inter-operator reproducibility $(\mathrm{kPa})$ & 1.4 & 0.7 & 0.8 & 0.8 & 0.6 & 0.5 & 0.7 & 0.5 & 0.4 & 0.3 & 1.0 \\
\hline Coefficient of variation $(\%)$ & 14 & & & 10 & 7 & 9 & 11 & 6 & 6 & 5 & 15 \\
\hline$p$ Value, at rest vs. stretched & $<10^{-3}$ & $<10^{-3}$ & $<10^{-3}$ & $<10^{-3}$ & $<10^{-3}$ & 0.013 & $<10^{-3}$ & $<10^{-3}$ & $<10^{-3}$ & $<10^{-3}$ & 0.059 \\
\hline
\end{tabular}

$\mathrm{BF}=$ biceps femoris; $\mathrm{GL}=$ gastrocnemius lateralis; $\mathrm{GM}=$ gastrocnemius medialis; $\mathrm{GRA}=$ gracilis; $\mathrm{RF}=$ rectus femoris; $\mathrm{SAR}=$ sartorius; $\mathrm{SM}=$ semimembranosus; $\mathrm{SOL}=$ soleus; $\mathrm{ST}=$ semitendinosus; $\mathrm{VL}=$ vastus lateralis; $\mathrm{VM}=$ vastus medialis.

the shear modulus, as the Young's modulus of fat is higher than that of muscle (Dubuis et al. 2012).

Bensamoun et al. (2006) have evaluated muscle shear modulus with magnetic resonance elastography at rest at $3.73 \pm 0.85 \mathrm{kPa}$ for $\mathrm{VL}, 3.91 \pm 1.15 \mathrm{kPa}$ for $\mathrm{VM}$ and $7.53 \pm 1.63 \mathrm{kPa}$ for SAR. These values are close to those measured in the present study (Table 2). An intraday coefficient of variation for two subjects was estimated at $19.4 \%$ for GL and $15.7 \%$ for biceps brachii by Ringleb et al. (2007). If intra-day variation is disregarded, SWE appeared slightly more reliable than magnetic resonance elastography, as noted by Lacourpaille et al. (2012).

There was no significant correlation between physiologic parameters (age, femur and tibia length, BMI, height and weight) and shear moduli of rested or stretched muscles. This result is in accordance with several previous studies that have found no correlation of shear modulus with physiological parameters (Aubry et al. 2013; Gennisson et al. 2005; Debernard et al. 2011), although correlations were observed between BMI and trapezius muscle stiffness (Kuo et al. 2013) and between BMI and muscle stiffness of the with biceps brachii and biceps femoris (Berko et al. 2014). However, as muscle shear modulus depends on a large number of parameters, the study of a small, specific and heterogeneous sample does not suffice to evaluate correlations; a large-scale study is needed.

A slight increase in muscle shear modulus was observed in preliminary measurements when subjects were supine with their legs hanging from the table; this phenomenon was eliminated by asking the subject to pause and walk between measurement sessions. In clinical routine, measurements can be performed in significantly shorter time, so the increase in shear modulus increase should not be an issue. A routine clinical measurement in a given muscle in two positions (relaxed and stretched) could last about $5 \mathrm{~min}$. This increase contrasts with the passive viscoelastic behavior of the muscle, so it was probably caused by a slight muscle contraction caused by discomfort. The viscoelastic behavior of muscle could potentially be further investigated with SWE (Gennisson et al. 2010).

The significant difference between measurements at rest and during passive stretching can be used to quantify differences between populations (healthy, athlete, pathologic, etc.). Work is in progress on the application of the protocol to subjects with neuromuscular diseases.

Shear wave elastography appears to be an appropriate tool for evaluation of the shear modulus of lower limb muscles, with good reproducibility, especially at rest, and the ability to characterize muscle properties both at rest and during passive stretching.

Acknowledgments-The authors are grateful to the ParisTech BiomecAM Chair Program on Subject-Specific Musculoskeletal Modeling for funding.

\section{REFERENCES}

Aubry S, Risson JR, Kastler A, Barbier-Brion B, Siliman G, Runge M, Kastler B. Biomechanical properties of the calcaneal tendon in vivo assessed by transient shear wave elastography. Skeletal Radiol 2013; 42:1143-1150. 
Basford JR, Jenkyn TR, An KN, Ehman RL, Heers G, Kaufman KR. Evaluation of healthy and diseased muscle with magnetic resonance elastography. Arch Phys Med Rehabil 2002;83:1530-1536.

Bensamoun SF, Ringleb SI, Littrell L, Chen Q, Brennan M, Ehman RL, An KN. Determination of thigh muscle stiffness using magnetic resonance elastography. J Magn Reson Imaging 2006;23:242-247.

Bensamoun SF, Ringleb SI, Chen Q, Ehman RL, An KN, Brennan M. Thigh muscle stiffness assessed with magnetic resonance elastography in hyperthyroid patients before and after medical treatment. J Magn Reson Imaging 2007;26:708-713.

Bercoff J, Tanter M, Fink M. Supersonic shear imaging: A new technique for soft tissue elasticity mapping. IEEE Trans Ultrason Ferroelectr Freq Control 2004;51:396-409.

Berko NS, Fitzgerald EF, Amaral TD, Payares M, Levin TL. Ultrasound elastography in children: Establishing the normal range of muscle elasticity. Pediatr Radiol 2014;44:158-163.

Botanlioglu H, Kantarci F, Kaynak G, Unal Y, Ertan S, Aydingoz O, Erginer R, Unlu M, Mihmanli I, Babacan M. Shear wave elastography properties of vastus lateralis and vastus medialis obliquus muscles in normal subjects and female patients with patellofemoral pain syndrome. Skeletal Radiol 2013;42:659-666.

Cornu C, Goubel F, Fardeau M. Muscle and joint elastic properties during elbow flexion in Duchenne muscular dystrophy. J Physiol 2001; 533:605-616.

Debernard L, Robert L, Charleux F, Bensamoun SF. Characterization of muscle architecture in children and adults using magnetic resonance elastography and ultrasound techniques. J Biomech 2011;44: 397-401.

Dubuis L, Avril S, Debayle J, Badel P. Identification of the material parameters of soft tissues in the compressed leg. Comput Methods Biomech Biomed Eng 2012;15:3-11.

Eby SF, Song P, Chen S, Chen Q, Greenleaf JF, An KN. Validation of shear wave elastography in skeletal muscle. J Biomech 2013;16: $2381-2387$.

Gennisson JL, Catheline S, Chaffai S, Fink M. Transient elastography in anisotropic medium: Application to the measurement of slow and fast shear wave speeds in muscles. J Acoust Soc Am 2003;114: $536-541$.

Gennisson JL, Cornu C, Catheline S, Fink M, Portero P. Human muscle hardness assessment during incremental isometric contraction using transient elastography. J Biomech 2005;38:1543-1550.

Gennisson JL, Deffieux T, Macé E, Montaldo G, Fink M, Tanter M. Viscoelastic and anisotropic mechanical properties of in vivo muscle tissue assessed by supersonic shear imaging. Ultrasound Med Biol 2010;36:789-801.

Hoang PD, Gorman RB, Todd G, Gandevia SC, Herbert RD. A new method for measuring passive length-tension properties of human gastrocnemius muscle in vivo. J Biomech 2005;38:1333-1341.

Hopkins WG. Measures of reliability in sports medicine and science. Sports Med 2000;30:1-15.
Hug F, Lacourpaille L, Maïsetti O, Nordez A. Slack length of gastrocnemius medialis and Achilles tendon occurs at different ankle angles. J Biomech 2013;46:2534-2538.

Koo TK, Guo JY, Cohen JH, Parker KJ. Relationship between shear elastic modulus and passive muscle force: An ex vivo study. J Biomech 2013;46:2053-2059.

Kot BCW, Zhang ZJ, Lee AWC, Leung VYF, Fu SN. Elastic modulus of muscle and tendon with shear wave ultrasound elastography: Variations with different technical settings. PLoS One 2012;7:e44348.

Kuo WH, Jian DW, Wang TG, Wang YC. Neck muscle stiffness quantified by sonoelastography is correlated with body mass index and chronic neck pain symptoms. Ultrasound Med Biol 2013;39: $1356-1361$.

Lacourpaille L, Hug F, Bouillard K, Hogrel JY, Nordez A. Supersonic shear imaging provides a reliable measurement of resting muscle shear elastic modulus. Physiol Meas 2012;33:19-28.

Lee WN, Larrat B, Pernot M, Tanter M. Ultrasound elastic tensor imaging: Comparison with MR diffusion tensor imaging in the myocardium. Phys Med Biol 2012;57:5075-5095.

Maiisetti O, Hug F, Bouillard K, Nordez A. Characterization of passive elastic properties of the human medial gastrocnemius muscle belly using supersonic shear imaging. J Biomech 2012;45:978-984.

Nordez A, Fouré A, Dombroski EW, Mariot JP, Cornu C, McNair PJ. Improvements to Hoang et al.'s method for measuring passive length-tension properties of human gastrocnemius muscle in vivo. J Biomech 2010;43:379-382.

Ringleb SI, Bensamoun SF, Chen Q, Manduca A, An KN, Ehman RL. Applications of magnetic resonance elastography to healthy and pathologic skeletal muscle. J Magn Reson Imaging 2007;25: 301-309.

Rouze NC, Wang MH, Palmeri ML, Nightingale KR. Finite element modeling of impulsive excitation and shear wave propagation in an incompressible, transversely isotropic medium. J Biomech 2013;46:2761-2768.

Royer D, Gennisson JL, Deffieux T, Tanter M. On the elasticity of transverse isotropic soft tissues. J Acoust Soc Am 2011;129:2757-2760.

Sarvazyan AP, Rudenko OV, Swanson SD, Fowlkes J, Emelianov SY. Shear wave elasticity imaging: A new ultrasonic technology of medical diagnostics. Ultrasound Med Biol 1998;24:1419-1435.

Shinohara M, Sabra K, Gennisson JL, Fink M, Tanter M. Real-time visualization of muscle stiffness distribution with ultrasound shear wave imaging during muscle contraction. Muscle Nerve 2010;42: 438-441.

Smith LR, Lee KS, Ward SR, Chambers HG, Lieber RL. Hamstring contractures in children with spastic cerebral palsy result from a stiffer extracellular matrix and increased in vivo sarcomere length. J Physiol 2011;589:2625-2639.

Wang M, Byram B, Palmeri M, Rouze N, Nightingale K. Imaging transverse isotropic properties of muscle by monitoring acoustic radiation force induced shear waves using a 2-D matrix ultrasound array. IEEE Trans Med Imaging 2013;32:1671-1684. 